\title{
Resolución de úlcera trocantérea mediante colgajo musculocutáneo de fascia lata en isla con cierre en V-Y
}

\author{
Trochanteric ulcer reconstruction by tensor fascia lata \\ musculocutaneous island flap with a $\mathrm{V}$ toY closure
}

Valentín A. Mogliani ${ }^{1}$, Andrea Arza ${ }^{1}$, Celeste Lorefice ${ }^{1}$, Francisco Olivero Vila²

\begin{abstract}
RESUMEN
Introducción. La úlcera trocantérea es una de las lesiones por presión más frecuente y está asociada generalmente a bursitis, por lo que su tratamiento es complejo.

Materiales y Métodos. Se presenta el caso de un paciente masculino de 29 años, con antecedente de lesión medular asociada a paraplejía fláccida con desarrollo de úlcera trocantérea derecha elíptica vertical. Fue abordado de forma multidisciplinaria y resuelto con resección de la patología y reconstrucción con colgajo musculocutáneo de fascia lata.

Resultado. El paciente fue dado de alta al 21 día posoperatorio. No presentó dehiscencias, infecciones de la herida ni recidiva al cumplirse 6 meses de la cirugía y el resultado estético y funcional fue satisfactorio.

Conclusión. El colgajo musculocutáneo de fascia lata ascendido y con cierre de $V$ en Y es una opción segura, versátil y reproducible para la resolución de úlceras trocantéreas. Sin embargo, deben respetarse todos los pasos de la técnica para evitar así complicaciones y recidivas.
\end{abstract}

Palabras claves: úlcera trocantérea, colgajo musculocutáneo de fascia lata.

\begin{abstract}
Background. Trochanteric ulcer is one of the most frequent pressure sore and is usually related with bursitis, therefore its treatment is complex.

Material and Methods. We present a case of a 29 year-old male patient with medical history of spinalcord injury (SCI) related to flaccid paraplegia and the development of right trochanteric vertical elliptical sore. He was treated by a multidisciplinary team and solved by the resection of the pathology and immediate reconstruction with tensor fascia lata (TFL) musculo cutaneous flap.

Results. The patient was discharged from the institution on the 21st postoperative day. With a follow-up of a 6 months period, complications such as wound infection, suture dehiscense or recurrence were not observed. Aesthetic and functional results were satisfactory.

Conclusion. Ascended TFL flap with a V-Y closure is a reproducible, reliable and versatile procedure for the coverage of trochanteric pressure sore defects. However all surgical techniques tages must be considered in order to avoid complications and recurrence of the disease.
\end{abstract}

Keywords: trochanteric ulcer, tensor fascia lata (TFL), musculocutaneous flap.

REVISTA ARGENTINA DE CIRUGÍA PLÁSTICA 2019;25(1):33-36. DOI/10.32825/RACP/201901/0033-0036

\section{INTRODUCCIÓN}

Las lesiones por presión (LPP) representan un importante problema de salud a nivel intra- y extrahospitalario y su prevalencia varía del 3 al 14\% en los pacientes hospitalizados ${ }^{1-3}$ y del 1,2 a $28 \%$ a nivel ambulatorio ${ }^{4}$. Las zonas más afectadas son la isquioperineal y trocantérea ${ }^{5-8}$. La presión en la región trocantérea se ejerce sobre el hueso recubierto por bursa, lo que provoca bursitis. Es por esto que se considera que estas lesiones se producen de la profundidad hacia la superficie y lo último en lesionarse es la piel (efecto iceberg) ${ }^{9}$. El tratamiento es complejo y requiere la resección del complejo úlcero-cicatrizal y de la bursa subyacente y la cobertura con colgajos.

1. Médico. Cursante de segundo año de la Carrera de Especialización en Cirugía Plástica de la Sociedad Argentina de Cirugía Plástica Estética y Reparadora (SACPER).

2. Médico. Cirujano Plástico. Jefe de Servicio de Cirugía Plásticadel IREP. Miembro Titular y Docente de SACPER. Servicio de Cirugía Plástica y Reconstructiva. Instituto de Rehabilitación Psicofísica (IREP). Ciudad Autónoma de Buenos Aires (CABA). Argentina

$\triangle$ Correspondencia: Dr. Valentín A. Mogliani. Tel. 549-3471-535553. valentinandresmogliani@gmail.com

Los autores no declaran conflictos de intereses
En 1978, Nahai et al. describieron el colgajo tipo I, con pedículo dominante único, musculocutáneo de tensor de fascia lata $(\mathrm{TFL})^{10}$. Este versátil colgajo es la alternativa de elección para el tratamiento de las úlceras por presión trocantéreas ${ }^{8,11}$. Sin embargo, hay consideraciones a tener en cuenta para garantizar su seguridad, evitar complicaciones y mejorar la estética y el resultado final.

El objetivo del trabajo es presentar y analizar, a propósito de un caso, las características del colgajo musculocutáneo de TFL ascendido con cierre de V en Y para resolución de úlcera trocantérea en el servicio de cirugía plástica del Instituto de Rehabilitación Psicofísica (IREP).

\section{PRESENTACIÓN DEL CASO}

Paciente masculino de 29 años, con antecedente de lesión medular en T4 secundaria a herida de arma de fuego en julio de 2016, asociada a paraplejía fláccida con desarrollo de LPP sacra y trocantérea derecha elíptica vertical, ambas con escara como forma de presentación. La úlcera sacra fue resuelta en nuestro servicio 5 días antes del caso a presentar. Previo a la cirugía, además de los estudios prequirúr- 


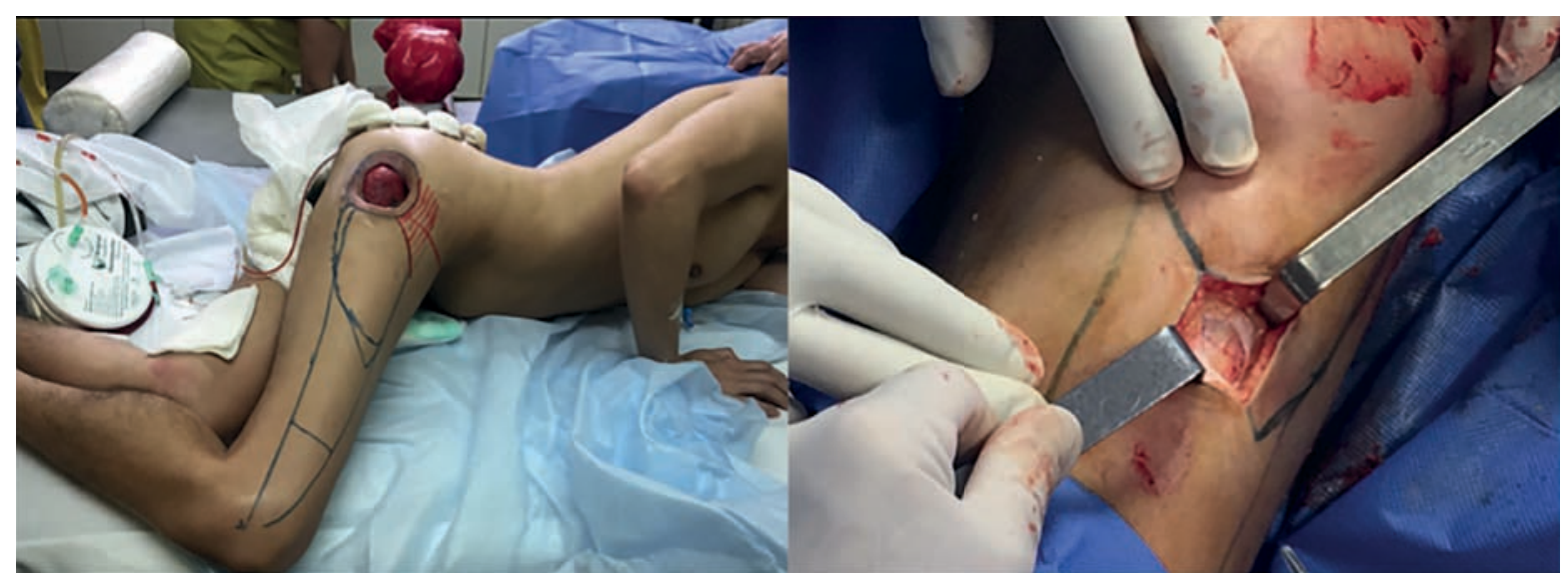

Figuras 1 y 2.

gicos de rutina y de la presencia de lesiones limpias, se requiere mantener un hematocrito de $30 \%$ y buen estado nutricional, tolerancia comprobada al decúbito ventral, disposición de 2 a 3 unidades de sangre y de un apropiado almohadón de sedestación. Se realizaron $\mathrm{Rx}$ panorámica de pelvis y tomografía de pelvis con reconstrucción en $3 \mathrm{D}$ que descartaron patologías asociadasy las correspondientes interconsultas con los servicios de fisiatría, urología e infectología del IREP.

\section{MATERIALES Y MÉTODOS}

\section{ANATOMÍA}

El territorio correspondiente a la fascia lata, de 8-9 x $30 \mathrm{~cm}$, está delimitado:

- Borde anterior: desde la espina ilíaca anterosuperior (EIAS) hasta la tuberosidad externa de la tibia.

- Borde posterior: desde el trocánter hasta la tuberosidad externa de la tibia.

- Ingreso del pedículo: arteria circunfleja femoral lateral, que ingresa al músculo a $10 \mathrm{~cm}$ distal a la EIAS, en contacto con el borde anterior del colgajo. El territorio distal de la fascia lata ( $5 \mathrm{~cm}$ distales) está irrigado por la arteria genicular y es poco seguro.

- Su inervación sensitiva proviene del nervio cutáneo femoral lateral, $1 \mathrm{~cm}$ por dentro y debajo de la EIAS. Su inervación motora procede de la rama inferior del nervio glúteo superior. Sin embargo, en estos pacientes lesionados medulares la sensibilidad es relativa ya que están denervados.

\section{TÉCNICA QUIRÚRGICA}

- Posición. Decúbito ventral sobre brete apoya pelvis.

- Marcación. Del complejo ulcerocicatrizal a resecar y del territorio correspondiente a la fascia lata de $8-9$ x $30 \mathrm{~cm}$. Se calcó el defecto a reparar (mediante una gasa) y se transportó y marcó el modelo en isla de piel contigua dentro del territorio de la fascia lata. Para facilitar el posterior cierre en v-y de la zona dadora, se marca una prolongación triangular distal (de vértice inferior) en la pastilla (Figura 1).
- Resección. De la úlcera, cicatriz periférica y bursa subyacente.

- Escoplado óseo. Aplanamiento de la superficie de apoyo hasta encontrar hueso macroscópicamente sano. Enviamos muestra a cultivo. No resecamos completamente el hueso para evitar una nueva LPP asociada a un cambio en el punto de apoyo. Hemostasia prolija ósea y de tejidos blandos.

- Tallado y disección del colgajo. Realizamos una pequeña incisión (ojal) en el extremo distalde la pastilla hasta observar e incidir la aponeurosis de la fascia lata, blanco nacarada (Figura 2). Comprobamos digitalmente, en el espacio retroaponeurótico, que los límites de la marcación coincidieran con el territorio anatómico real del músculo (Figura 3). Se continuó el tallado de la unidad musculocutánea de distal a proximal primero por el borde posterior, que puede ser incidido hasta la úlcera, y luego por el borde anterior, en el que el músculo se secciona hasta $10 \mathrm{~cm}$ de la EIAS para respetar el pedículo. En el borde anterosuperior realizamos disección subcutánea amplia para permitir el ascenso del colgajo y facilitar el cierre sin tensión (Figuras 4, 5 y $\mathbf{6}$ ).

- Movilización. En forma ascendente vertical (Figura 7).

- Colocación de drenaje. Con doble tubuladura para la zona dadora y el colgajo.

- Cierre. Sin tensión de zona dadora y receptora en V-Y. En dos planos y con puntos separados (Figura 8).

- Apósito de Spadafora-Durand. En zona dadora y receptora. Evita colecciones, mejora el retorno venoso y relaja la tensión de las suturas (Figura 8).

\section{CONSIDERACIONES DE LA TÉCNICA}

El espacio retroaponeurótico, al que se accede para la comprobación digital de los límites del colgajo, es avascular y virtual y presenta un repliegue que marca los límites anterior y posterior del músculo. Si la marcación de la pastilla de piel supera los límites anatómicos reales tiene alto riesgo de sufrimiento, por lo que debe corregirse en este momento para garantizar una buena irrigación del colgajo. 


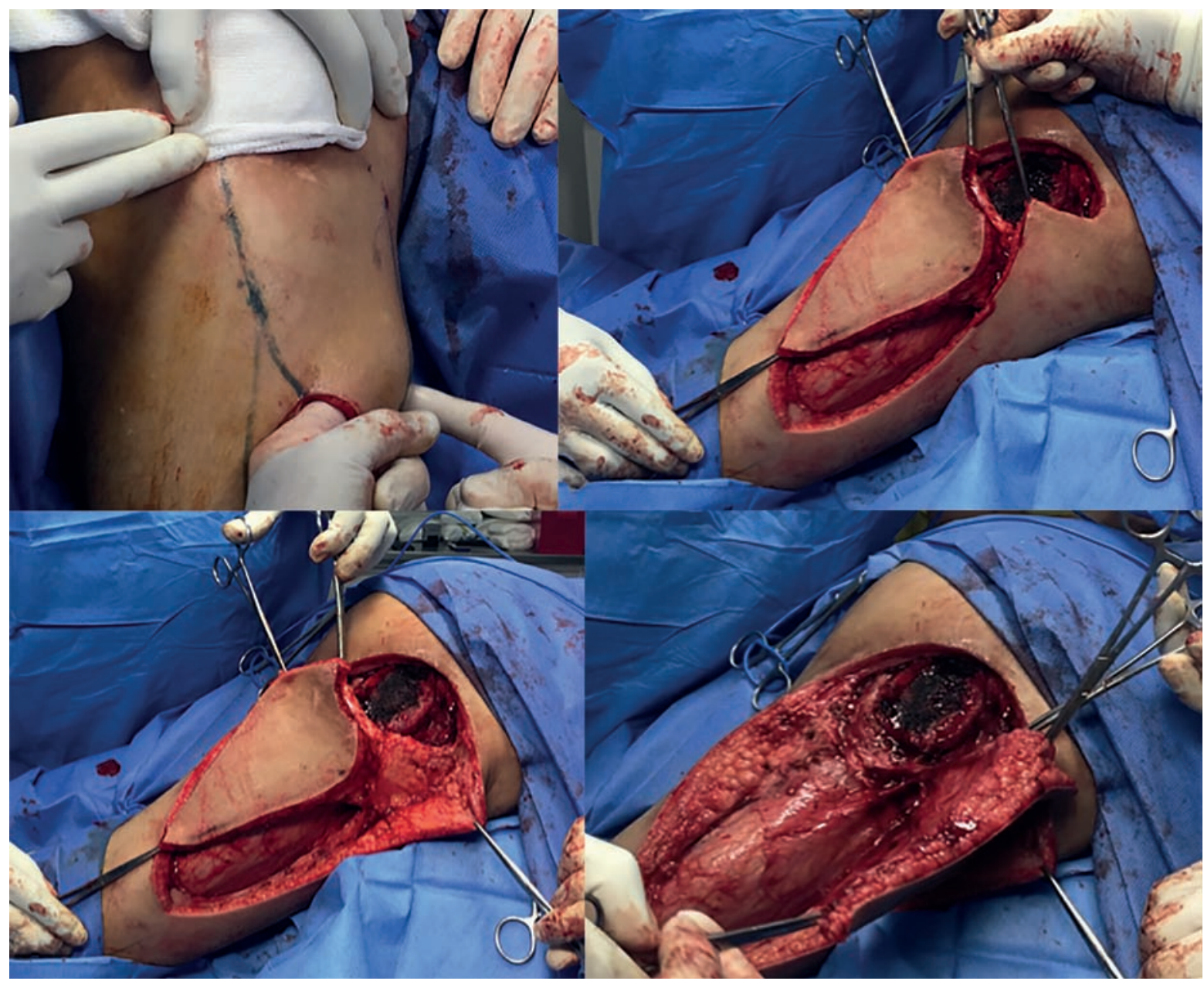

Figuras 3, 4, 5 y 6.

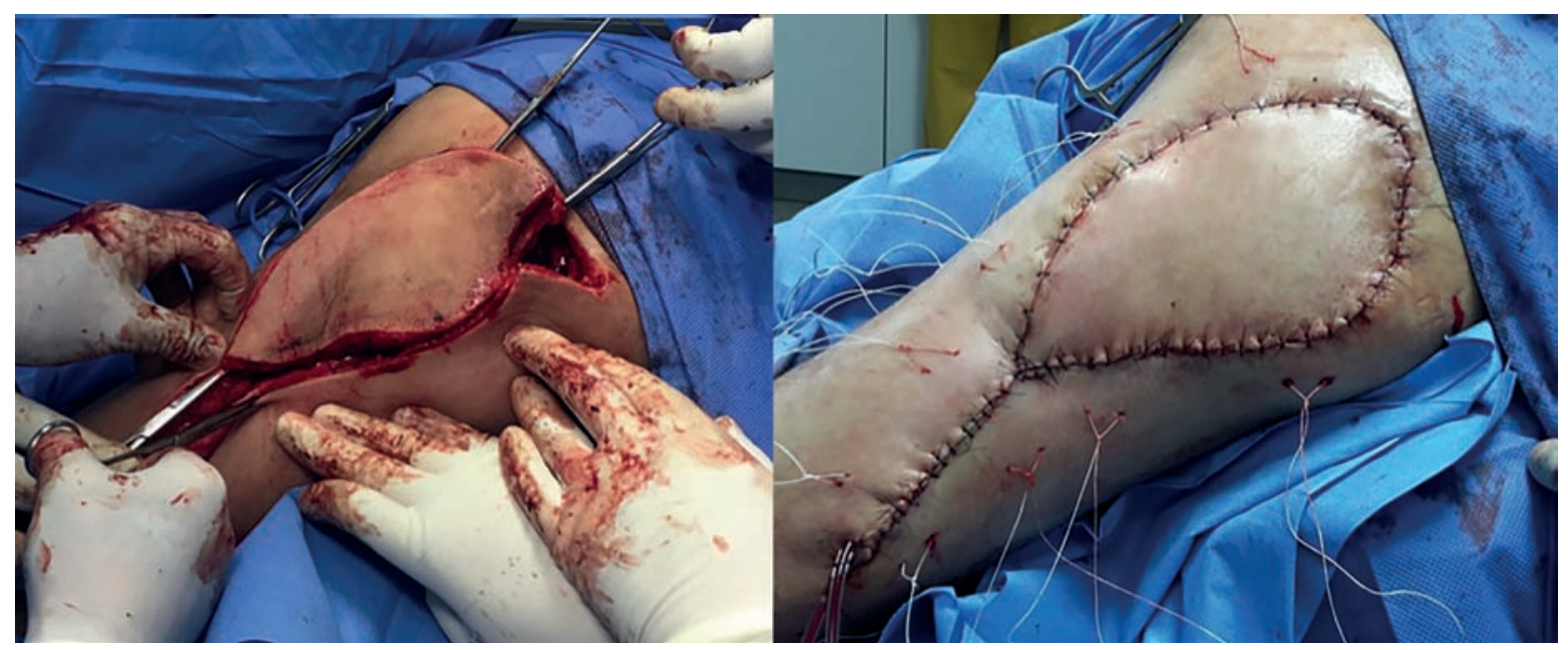

Figuras 7 y 8 .

CONTROLES POSOPERATORIOS Y RESULTADOS Se transfundió 1 UGRD en el posoperatorio inmediato y otra a las 48 hs para mantener un hematocrito de 30\% y garantizar la vitalidad del colgajo. Se realizó la primera curación y se retiraron los drenajes aspirativos al 4to día posoperatorio (débito menor a $10 \mathrm{ml}$ en $48 \mathrm{hs}$ ). Al 10mo día comenzamos con curaciones expuestas (retirando el apósito de Spadafora-Durand). El paciente permaneció en decúbito ventral estricto por 20 días, fue dado de alta al 21 día posoperatorio e instruido para apoyar progresivamente la zona operadaa partir del día 30.

El cultivo del escoplado óseo fue (-). El paciente no presentó complicaciones como dehiscencias o infecciones de la herida ni recidivas y el resultado estético y funcional fue satisfactorio al cumplirse 6 meses de la cirugía (Figura 9). 


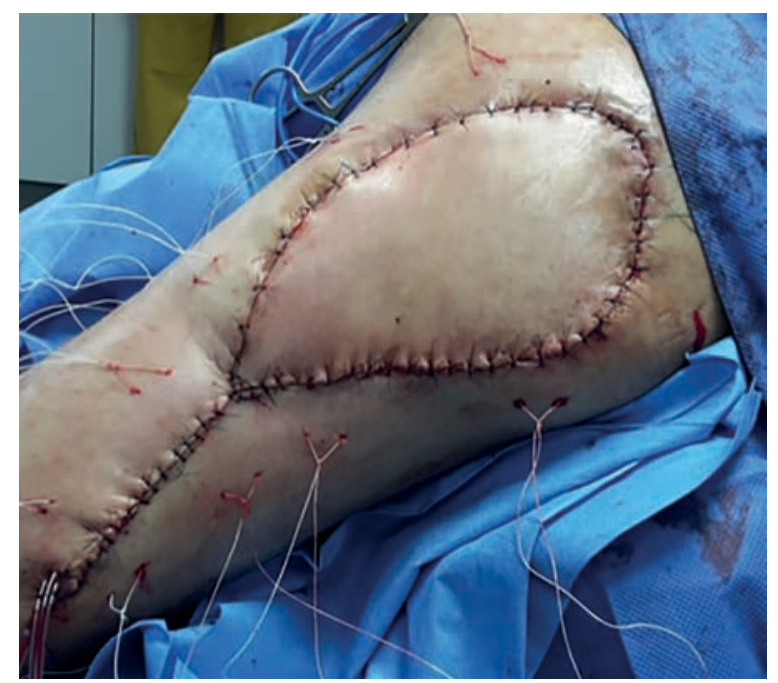

Figura 9.

\section{DISCUSIÓN}

Son varios los trabajos que proponen el colgajo de TFL como el gold standard en la reparación de úlceras trocantéreas ${ }^{8,11}$; sin embargo, existen muchas variables y no todas respetan los pasos de la cirugía, desde la selección y preparación del paciente hasta las consideraciones técnicas intraoperatorias y los cuidados posoperatorios.

Algunas de las complicaciones más frecuentemente reportadas son seromas, dehiscencias de suturas y sufrimiento de la punta ${ }^{8}$. Proponemos, según nuestra experiencia, el empleo del apósito de Spadafora-Durand como una técnica útil para prevenir estas complicaciones.
Calcar el defecto a reparar en forma de isla dentro de la superficie de la fascia lata y comprobar los límites de la marcación mediante maniobra digital retroaponeurótica luego de realizar el ojal, son gestos técnicos sencillos que disminuyen el riesgo de sufrimiento de la pastilla.

Una amplia disección subcutánea a nivel de la región anterosuperior del territorio de la fascia lata, no sólo permite el ascenso sin tracción de la pastilla a movilizar sino que además facilita el cierre sin tensión de la zona dadora. Esta maniobra no conlleva riesgo de lesionar el pedículo ya que el mismo ingresa al colgajo por la cara profunda del músculo.

Esta técnica, presentada en el año 2001 por el IREP en el Congreso Argentino de Cirugía Plástica y premiada en dos oportunidades por la SACPER, es una opción segura, versátil y reproducible para la resolución de úlceras trocantéreas de cualquier tamaño y previene secuelas funcionales y estéticas importantes en la zona dadora ya que el cierre en V-Y evita injertos. Sin embargo, deben respetarse todos los pasos de la técnica para evitar así complicaciones y recidivas.

\section{AGRADECIMIENTOS}

A los servicios de fisiatría, infectología, urología, traumatología y enfermería del IREP por su compromiso diario en el trabajo multidisciplinario que requiere el abordaje de los pacientes lesionados medulares.

$\mathrm{Al}$ servicio de cirugía plástica del IREP, por su participación permanente en el desarrollo de cirujanos plásticos en formación.

\section{BIBLIOGRAFÍA}

1. Bauer J, Mancoll JS, Phillips L. Pressure Sore. Grabb \& Smith PlasticSurgery 6th Edition Lippincott, Williams \& Wilkins. Philadelphia, 2007.Pp: 722-9.

2. Ishida L, Munhoz A, Montag E, et al.Tensor Fasciae Latae Perforator Flap: Minimizing Donor-Site Morbidity in The Treatment of Trochanteric Pressure Sores. Plast Rec Surg 2005:116:1346.

3. Paletta CE, Freedman B, Shehadi SI. The Tensor Fasciae Latae Musculocutaneous Flap. Plast Rec Surg 1989;83:852.

4. Lynch SM. The Bilobed Tensor Fasciae Latae Myocutaneo is Flap. Plast Rec Surg 1981;67:796.

5. Yohena R, Olivero Vila F, et al. Lesiones por presión. En: Coiffman F. Cirugía plástica, reconstructiva y estética. Caracas, Ed. Amolca; 2016. TomoVI. Cap.191. p. 2385-6.

6. Yohena $R$, Olivero Vila F, et al. Lesiones por presión. En: Coiffman F. Cirugía plástica, reconstructivayestética. Caracas, Ed. Amolca; 2016. TomoVI. Cap. 191.p. 2392-3.
7. Diaz S, Li X, Rodriguez L, Salgado C. Update in the Surgical Management of Decubitus Ulcers. Anaplastology 2013.

8. Calderón W, Oyarse E, et al. Colgajo en hacha de tensor de fascia lata paraúlceras por presión trocantéreas. Cir plást iberolatinoam. Vol.36 No 4 Octubre-Noviembre-Diciembre 2010. Pag. 355-8.

9. Yohena R, Olivero Vila F, et al. Lesiones por presión. En: Coiffman F. Cirugía plástica, reconstructiva y estética. Caracas, Ed. Amolca; 2016. TomoVI. Cap. 191.p. 2388.

10. Nahai F, Silverton JS, Hill HL, Vasconez LO. The Tensor Fascia Lata Musculocutaneous Flap. Ann Plast Surg 1978;1:371-9.

11. Alvarez D, Colicchio O, Galvao $R$, Zuccolotto $P . V$ - $Y$ tensor fascia lata for trochanteric pressure sore treatment. Can J Plast Surg 1997:5(4):213-6. 